\title{
Cost-Benefit Analysis of Unreliable System Protection Scheme Operation
}

\author{
José Luis Calvo de Miguel \\ Pedro Javier Ramírez \\ Simon H. Tindemans \\ Goran Strbac \\ Department of Electrical and Electronic Engineering \\ Imperial College London \\ London, United Kingdom
}

\begin{abstract}
System Protection Schemes (SPS) have the potential to greatly enhance the utilization of the network, often by automatically disconnecting generators in response to contingency events. However, malfunctions of such systems may expose the system to harmful blackouts. The operation of unreliable SPS is therefore subject to a cost-benefit balance between the benefits of increased system utilization and the risk of outages. This paper studies this trade-off in a year-round basis. The problem is firstly stated from a centralized perspective to probabilistically minimize the operational costs for a whole operating year. A case study based on a basic simple representation of the Great Britain system is considered. The results show great annual benefits from equipping the SPS with multiple generation disconnection systems, which are mainly associated with critical operating conditions. However, it is demonstrated that redundant SPS configurations do not necessarily reduce the levels of operational risk exposure.
\end{abstract}

Index Terms-Cost-benefit analysis, probabilistic security standards, frisk assessment, system protection schemes

\section{INTRODUCTION}

$\mathrm{T}$ HE so-called SPS/SIPS/RAS (System Protection Scheme/System Integrity Protection Schemes/Remedial Action Scheme) are protection systems designed for detecting abnormal operating system conditions, typically contingency-related, and for initiating pre-planned corrective actions to mitigate the consequences of the abnormal condition. SPS actions rely on ICT infrastructure and typically include automatic generation disconnections, which are commonly known as generation rejection schemes [1] or intertripping schemes [2]. These corrective schemes allow system operators to keep acceptable system stability and power flows in postcontingency scenarios without requiring to pre-constrain the network. This concept is opposed to the traditional deterministic ' $\mathrm{N}-2$ ' security standard, which requires large transmission capacity margins in order to withstand the loss of one or two circuits without threatening the power system operation stability. As such, SPS have greatly facilitated the application of probabilistic security standards which seek to balance benefits and risks associated with different levels of network utilization and investment [3].

The potential benefits of SPS are especially important when large volumes of remote wind power generation are connected to the grid. In operational timescales SPS reduce the need for wind curtailment, which translates into system operating costs reductions. In the long term SPS defer costly transmission investments associated with wind generation projects [4]. As a result, there has been a growing interest in the use of SPS in order to facilitate a cost-effective integration of renewable generation. The Great Britain (GB) system is an example of a power system with remote renewable generation, as large volumes of wind power generation are connected in Scotland, while the demand is mostly concentrated in England. The Scotland-England transmission corridor consists of two AC double circuits, which will be complemented with two offshore HVDC lines, in order to increase the North-South power transfer [5]. However, National Grid -the GB system operatorcould arrange commercial intertripping scheme contracts in order to enhance the utilization of this zone of the network [2].

Obviously, the operation of SPS is not $100 \%$ reliable, and their utilization may expose the system to additional risks resulting from SPS malfunction. Ultimately, SPS failures may largely destabilize the system, resulting in harmful blackouts. In a survey conducted by IEEE-CIGRE in 1996 [6], stakeholders from the power industry assigned the cost from SPS failures to the highest cost category. SPS malfunctions have definitely contributed to cascading outages during the last decade, as happened in the Nordic network in 2005 [1]. Furthermore, SPS malfunctions have historically been more frequent than one could expect. For example, the NERC System Disturbance Reports from 1986-1995 [1] indicate that from the 30 cases that involved the operation of SPS, 21 were reported as successful operation of SPSs, while 9 involved operational failures. Therefore, the increasing use of SPS demands a better understanding of their impact, since undesirable SPS operations may result in the deterioration of the overall system reliability.

A cost-benefit analysis of system protection scheme operations, based on a simple representation of the GB system, is presented in this paper. The analysis considers a set of representative operating conditions across the year, and the possibility for the system operator to arrange various intertripping schemes, at the expense of an annual capability fee. The problem is formulated from a centralized perspective to probabilistically minimize the intertripping capability fee payments, and the annual generation, protection and load curtailment costs for a whole operating year. Our case study shows that equipping the SPS with multiple intertripping schemes is associated with important annual benefits, especially when compared to system operations under the traditional ' $\mathrm{N}-2$ ' security standard. The analysis shows that the 
main operational benefits are linked to stressed operating conditions characterized by bad weather and high demand and availability of wind outputs. It could be expected that contracting more intertripping schemes would reduce the levels of operational risk exposure; however, the inspection of the different cost components reveals that this is not necessarily the case within the probabilistic security framework considered in this paper.

\section{PROBLEM STATEMENT}

Each year, the system operator solves a probabilistic optimization problem that seeks to minimize the total annual operating cost by arranging a number of intertripping scheme contracts with generating units. The problem has three types of operational decisions: 1 ) the number $N$ of generating units that will be under intertripping contracts for a year of operation; and for each operating condition $k: 2$ ) the dispatch of generators $\mathcal{D}_{k}$ and, 3 ) the operational configuration of the SPS, i.e. $\mathcal{S}_{k}$. The objective function of the problem solved by the system operator can be written then as follows:

$$
\min _{\mathrm{N}, \mathcal{D}, \mathcal{S}} C+\sum_{k \in \mathcal{K}}\left(G_{k}+P_{k}^{a}+\sum_{c \in \mathcal{C}} \sum_{o \in \mathcal{O}} \lambda_{k, c} p_{o}\left(P_{k, c, o}^{u}+X_{k, c, o}\right)\right)
$$

The constraints of the optimization problem are given by:

$$
\begin{gathered}
h_{k}\left(\mathcal{D}_{k}, \mathcal{S}_{k}\right) \leq 0, \quad \forall k \in \mathcal{K}, \\
g_{k}\left(\mathcal{D}_{k}, \mathcal{S}_{k}\right)=0, \quad \forall k \in \mathcal{K}, \\
\mathcal{S}_{k} \in \Sigma\left(\mathrm{N}, \mathcal{D}_{k}\right), \quad \forall k \in \mathcal{K} .
\end{gathered}
$$

The objective function (1) includes the annual capability cost of the intertripping contracts $(C(N)$, first term), and the costs associated with the different system operation conditions (second to fourth term). The costs associated with each operating condition $k$ are given by: a) the conventional generation cost $\left(G_{k}\left(\mathcal{D}_{k}, \mathcal{S}_{k}\right)\right.$, second term); b) the availability fee of the protection services $\left(P_{k}^{a}\left(\mathcal{D}_{k}, \mathcal{S}_{k}\right)\right.$, third term); and, c) the fourth term that represents the expected value of the costs from contingencies. The costs associated to contingencies is a summation over all the possible contingencies $c \in \mathcal{C}$, weighted by their respective fault rates $\lambda_{k, c}$, followed by a summation over all the possible SPS outcomes $o \in \mathcal{O}(c, \mathcal{S})$ (including malfunctions), which are weighted by the probability of occurrence $p_{o}$ of each SPS outcome. For each contingencySPS-outcome pair, the utilization costs $P_{k, c, o}^{u}$ and load-shedding impact $X_{k, c, o}$ are included. Each operating condition is multiplied by $h_{k}$, which is the number of hours that such condition is expected to happen during the year. The optimization is subject to inequality and equality constraints on the power flows and the generation dispatch, i.e. constraints (2) and (3) respectively [7]. The last set of constraints (4) enforces that the SPS configurations in operational timescales are compatible with the intertripping schemes annually contracted and with the dispatch of generators.

\section{CASE STUDY}

In this paper the key elements of a cost-benefit model are mentioned. The full model will be explained somewhere else.

\section{A. Description}

Figure 1 illustrates the power system under consideration, which is a simplified representation of the GB system. The wind power generation is connected in North (Scotland), while the electricity demand is mostly concentrated in South (England). The power flows downstream through two identical double circuits, each divided in two sections. The representation then has a total of eight identical lines $\left(\mathrm{L}_{1} \ldots \mathrm{L}_{8}\right)$, each with a circuit rating of $1700 \mathrm{MW}$ [4]. The system is assumed to have ten wind farms of $2 \mathrm{GW}$ each as shown in Fig. 1. Under the traditional ' $\mathrm{N}-2$ ' security standard, the transmission system should not be overloaded in case of losing two transmission lines. Such scenario constrains the power transfer to $50 \%$ of the available transmission capacity.

In order to allow power transfers beyond the ' $\mathrm{N}-2$ ' limit, it is assumed that an SPS is installed. Upon the occurrence of a line contingency, the SPS disconnects the faulty lines and a preprogrammed amount of wind generation in North, in order to reduce the power flows and thus avoid overloading the remaining transmission lines. A probabilistic security standard is assumed, therefore the power transfer is increased up to the point that the cost (including risk from the SPS malfunctions) associated with such levels of utilization exceeds the benefits.

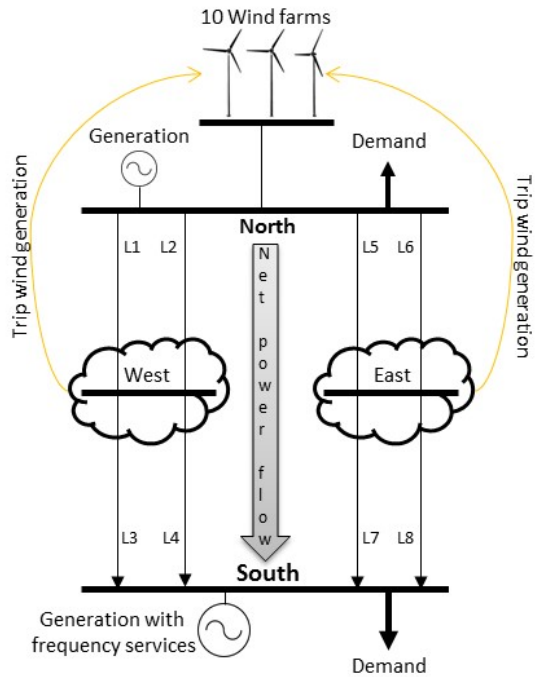

Fig. 1. 4-bus representation of the GB power system.

An operating year is represented by a set $\mathcal{K}$ of 200 representative operating conditions [8]. Each operating condition $k$ is characterized by: a) a global demand factor $(\%$ of system peak demand) and available wind output factor ( $\%$ of peak wind generation); b) weather conditions (good or bad); and, c) the number of hours during which each operating condition occurs over the year. It is assumed that in each operating condition the global available wind output is equally distributed across the ten wind farms.

The system operator, as discussed in the previous section, solves the optimization problem described by equations (1) to (4). The system operator thus arranges a number of yearly individual intertripping contracts with the available wind farms located in North (i.e. $N=1,2, \ldots, 10$ ), which are consequently connected to the system protection scheme, at the expense of an annual capability fee. As a result, the system operator is able 
to select wind farms throughout the year from those awarded with the intertripping agreement.

\section{B. SPS failure and impact modelling}

As illustrated in Fig.2, the SPS is connected to the eight transmission lines of the corridor, and to the $N$ wind farms under the intertripping agreement. The system can be triggered by single and double circuit contingencies. In operational timescales the system operator selects a number of wind farms, i.e. $n$, that are expected to be disconnected for any of these triggering events, being $n \leq N$. It is assumed that the system operator assigns an intertripping capacity $s$ evenly amongst the selected wind farms. Therefore, in operational timescales the configuration of the SPS is defined by the number of wind farms selected and the intertripping capacity assigned to them i.e. $\mathcal{S} \equiv(s, n)$.

The relays, circuit breakers and logic controls can fail according to a hidden failure model [9] which results in an average availability of $0.981,0.9925$ and 0.998 , respectively. Additionally, the communication links are assumed to have an average availability of $95 \%$. The unavailability of these components to perform their function results in an abnormal operation of the SPS. For instance, the malfunction of the communication channel associated with one of the $n$ selected generators results in a loss of a fraction $(1 / n)$ of the scheduled intertripping capacity $s$.

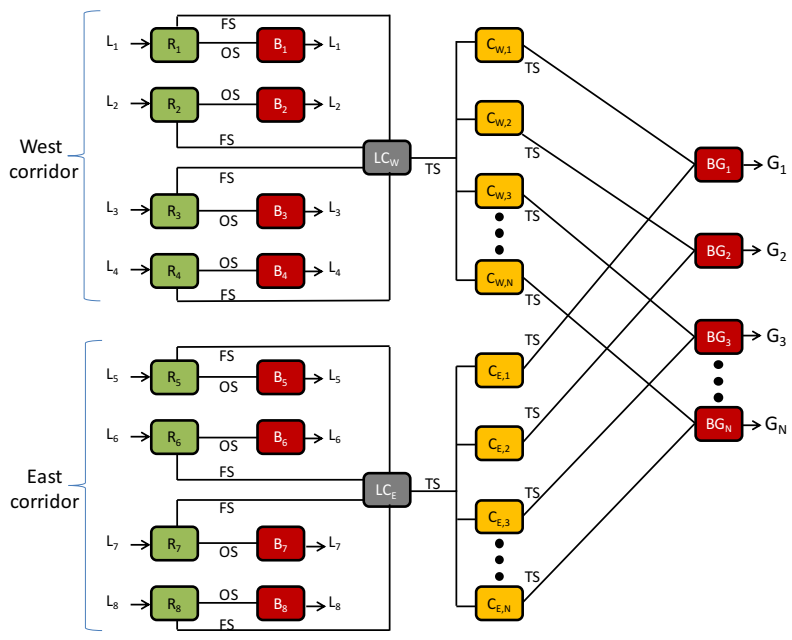

Fig. 2. Connection diagram of the SPS components. Relays (connected to transmission lines) are in green, breakers in red, control in grey, and communication channels in yellow.

As single and double line contingencies are considered, the SPS outcomes are characterized by the loss of one or two lines (local actions to isolate the faulty lines), as well as by the number of generators that are successfully tripped (remote actions to reduce power flows). The local result is represented by the label $l \in\{1,2\}$, denoting single or double circuit outages respectively. The remote actions are represented by the number $m(m \in\{0, \ldots, n\})$ of generators that were successfully tripped. As the SPS can be triggered by single and double line faults, the outcomes are conditional on those events, which result in conditional probabilities $\left(p_{m / n}^{l} \mid e\right)$ for an initiating event $e \in$ $\left\{e_{s}, e_{d}\right\}$ (single or double line fault). The values of these probabilities depend on the number of wind farms selected, i.e.n, and therefore they are internally computed by the optimization model described in the next section.

The operation of the SPS results in a generation shortage. Then frequency-sensitive generators located in South restore the power balance within seconds. After the generationdemand balance is restored, the power is re-routed over the operational transmission lines. If the post-SPS power flow exceeds the remaining capacity of the transmission corridor (5100 MW and $3400 \mathrm{MW}$ for a single outage and a double outage respectively), the disconnection of the remaining lines is triggered, which isolates North from South (system splitting) and requires a second restoration in order to balance generation and demand within each zone. Such situation causes large involuntary load shedding in South.

\section{Optimization model}

As stated in section II, the optimization model considers multiple operating conditions for addressing the operation of the power system in a year-round basis. This model is solved in Fico Xpress. The objective function is given by:

$$
\min _{N, u_{i}, g_{i}, \Delta g_{i}, r, s, n} C+\sum_{k}\left(G_{k}+P_{k}+X_{k}\right) \times h_{k},
$$

which is subject to the definitions and constraints listed below.

\section{1) SPS capability}

The SPS capability cost is computed as the number of annual intertripping contracts $N$ times a yearly capability fee $\pi^{y}$ of of $£ 70000 /$ year:

\section{2) Generation}

$$
C \equiv C(\{N\})=\pi^{y \cdot N},
$$

The generation cost [4] $G_{k}$ is as a linear function of the dispatched output, i.e. $g_{k, i}$, of each generator $i$ :

$$
G_{k} \equiv G_{k}\left(\left\{g_{k, i}\right\}\right)=\sum_{i} \alpha_{i} g_{k, i}
$$

The dispatched generating capacity should equal the total demand in North, i.e. $D_{N}$, and South, i.e. $D_{S}$, nodes:

$$
\sum_{i} g_{k, i}=D_{k}^{N}+D_{k}^{S} \text {. }
$$

The North-South flow $t_{k}$ is subject to the maximum flow $\bar{t}$ :

$$
t_{k}=\sum_{\substack{i \in \text { North } \\ t_{k} \leq \bar{t} .}} g_{k, i}-D_{k}^{N},
$$

The commitment of generating units is modelled as follows:

$$
\begin{gathered}
g_{k, i} \geq \underline{g}_{i} u_{k, i}, \\
g_{k, i}+\Delta g_{k, i} \leq \bar{g}_{i} u_{k, i} .
\end{gathered}
$$

where $u_{i}$ represents the binary commitment status of each generator and $\Delta g_{i}$ the reserve capability from part-loaded generators in South constrained by:

$$
\begin{gathered}
0 \leq \Delta g_{k, i} \leq \overline{\Delta g}_{i}, \quad \forall i \in \text { South } \\
\Delta g_{k, i}=0, \quad \forall i \in \text { North }, \\
\sum_{i} \Delta g_{k, i}=r \\
r_{k} \geq 1800 \mathrm{MW} .
\end{gathered}
$$

The minimum value of the reserve $r_{k}$ is $1800 \mathrm{MW}$, but the system operator may decide to contract additional intertripping 
and reserve capacity if this is beneficial for reducing the total system costs. The additional capacity may be used to relax constraints on the intertripping capacity $s_{k}$, given by:

\section{3) System protection}

$$
0 \leq s_{k} \leq r_{k} \text {. }
$$

In the second term of the objective function (5), $P$ denotes the expected hourly cost of system protection services, consisting of frequency response, and SPS availability and utilisation fees. The availability cost is determined in advance by purchasing a response capacity $r_{k}$ at a fee $\pi_{a}$ of $£ 30 / \mathrm{MW} / \mathrm{h}$. The actual SPS utilisation fees are unknown, as they depend on the stochastic occurrence of outages and the actual amount of generation tripped in response to these outages. Successfully tripped generation capacity is compensated with an utilisation fee $\pi_{u}$ of $£ 1000 / \mathrm{MW} /$ event

$\lambda_{s}$ and $\lambda_{d}$ are the rate of single circuit fault events in any one of the eight lines (occurrences/hour), and the common rate of double circuit fault events (occurrences/hour) in lines that are connected to the SPS on the same side of the intermediate buses $\left(\mathrm{L}_{1}\right.$ and $\mathrm{L}_{2}, \mathrm{~L}_{3}$ and $\mathrm{L}_{4}, \mathrm{~L}_{5}$ and $\mathrm{L}_{6}, \mathrm{~L}_{7}$ and $\mathrm{L}_{8}$ ), respectively. Their values depend on the weather conditions, as shown in Table I. The total rates (occurrences/hour) for each type of occurrence are then given by $\lambda_{e_{s}}^{\text {tot }}=8 \lambda_{s}$ and $\lambda_{e_{d}}^{\text {tot }}=4 \lambda_{d}$. Then, the system protection services associated with contingency $k$ can me calculated as follows:

$$
\begin{gathered}
P_{k} \equiv P_{k}\left(r_{k}, s_{k}, n_{k}\right)= \\
\pi^{a} \cdot r_{k}+\pi^{u} \cdot s_{k} \sum_{\substack{e \in\left\{e_{s}, e_{d}\right\} \\
\cdot p_{m / n}^{l} \mid e}} \lambda_{e}^{t o t} \sum_{l \in\{s, d\}} \sum_{m \in\{0 \ldots n\}} \frac{m}{n_{k}} .
\end{gathered}
$$

TABLE I.

FAULT RATES PROBABILITIES

\begin{tabular}{|c|c|c|}
\hline Weather scenario & Outage order & Fault rate (occ/h) \\
\hline \multirow{2}{*}{ fair } & single & $3 \times 10^{-5}$ \\
\cline { 2 - 3 } & double & $3 \times 10^{-6}$ \\
\hline \multirow{2}{*}{ bad } & single & $1 \times 10^{-3}$ \\
\cline { 2 - 3 } & double & $1 \times 10^{-4}$ \\
\hline
\end{tabular}

$$
\text { Fault rates based on data from [4] }
$$

The intertripping capacity assigned to a wind farm cannot be larger than its actual wind availability. As it is assumed that the total available wind output is equally distributed amongst the ten sites in the system, the previous can be expressed as follows:

\section{4) Risk}

$$
s_{k} \leq n_{k} \times w_{k} \times 2 G W
$$

The term $X_{k}$ in the objective function represents the expected cost resulting from emergency load shedding, i.e. the risk. The risk is computed as the price of emergency customer disconnections, $\pi^{x}=£ 30000 / M W /$ event, multiplied by the frequency of occurrence and the expected amount of load shed. The previous can be expressed as follows:

$$
\begin{aligned}
& X_{k} \equiv X_{k}\left(t_{k}, r_{k}, s_{k}, n_{k}\right)= \\
& \pi^{x} \sum_{e \in\left\{e_{s}, e_{d}\right\}} \lambda_{e}^{t o t} \sum_{l \in\{s, d\}} \sum_{m \in\{0 \ldots n\}} p_{m / n}^{l} \mid e \\
& \text { - } \Delta D_{k, l, m}\left(t_{k}, r_{k}, s_{k}, n_{k}\right) \text {. }
\end{aligned}
$$

$\Delta D_{k, l, m}\left(t_{k}, r_{k}, s_{k}, n_{k}\right)$ denotes emergency load shedding (MW) as a consequence of system splitting. It is computed using binary auxiliary variables, i.e. $\delta_{k, l, m}$, that reflects the presence of post-SPS overload conditions and $\eta_{k}$ that has the value 1 only if $\left(t_{k}-r_{k}\right)$ is positive. The value of $\Delta D_{k, l, m}\left(t_{k}, r_{k}, s_{k}, n_{k}\right)$ is determined by the following set of equations:

$$
\begin{aligned}
& t_{k}-\frac{m}{n} s_{k}-\bar{t}_{l} \leq \delta_{k, l, m} M, \quad \forall k, l, m_{1} \text { (21) } \\
& t_{k}-\frac{m}{n} s-\bar{t}_{l} \geq-\left(1-\delta_{k, l, m}\right) M, \quad \forall k, l, m \\
& t_{k}-r_{k} \leq \eta_{k} K \\
& t_{k}-r_{k} \geq-\left(1-\eta_{k}\right) M \text {, } \\
& \Delta D_{k, l, m} \geq 0 \text {, } \\
& \Delta D_{k, l, m} \leq \delta_{k, l, m} M \text {, } \\
& \Delta D_{k, l, m} \leq \eta_{k} M \text {, } \\
& \Delta D_{k, l, m} \geq t_{k}-r_{k}-\left(2-\delta_{k, l, m}-\eta_{k}\right) M, \quad \forall k, l, m_{1} \\
& \Delta D_{k, l, m} \leq t_{k}-r_{k}+\left(1-\eta_{k}\right) M,
\end{aligned}
$$

where $K$ is a large positive number (constant). It must be noted that the optimisation problem given by equations (5) to (29) is non-linear due to the appearance of $n_{k}$ in the denominators of (21) and (22). Because the number of wind farms of the case study is small i.e. $N=1, \ldots, 10$, equations (5)-(29) are solved independently for each possible value of $N$ and $n_{k} \leq N$ and the best solution is selected.

\section{Results and discussion}

\section{1) Optimal number of intertripping contracts}

The following discussion is focused on the analysis of the system operational costs as a function of the annual number of intertripping contracts.
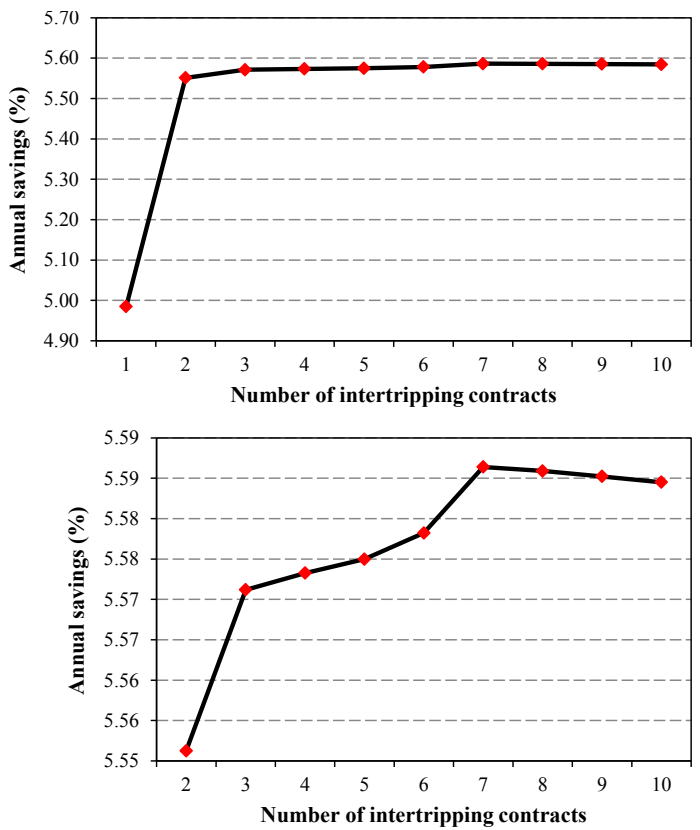

Fig. 3. Percentage of annual savings as a function of the number of intertrippings contracted with respect to ' $\mathrm{N}-2$ ' case. The lower graph is an enlargement for $N=2, \ldots, 10$ only.

In the base case scenario the system operator is subject to the traditional ' $\mathrm{N}-2$ ' security standard and does not use the SPS. The total annual operational costs of the power system amount to $£ 8391.4$ million. Figure 3 shows the percentage of 
annual savings with respect to the base case scenario for the different number of possible intertripping contracts. The bottom plot in Figure 3 corresponds to a zoom of the top plot for $N \geq 2$. If the system operator decides to contract intertripping services with only one wind farm, the total annual system costs are reduced by $4.98 \%$ with respect to the base case scenario. In absolute terms, this translates into operational savings of about $£ 417.9$ million. The second intertripping contract provides extra savings of approximately $0.57 \%$, which achieves savings of $£ 462.36$ million in absolute terms. Additional intertripping contracts continue to report further annual savings (approximately $0.007 \%$ of savings per contract in average), until the seventh contract, where the tendency changes and the total annual costs increase with additional intertripping arrangements. The optimal solution is therefore the acquisition of intertripping services from 7 wind farms. The total annual savings with respect to the ' $\mathrm{N}-2$ ' base case amount to $£ 468.8$ million.

Figure 4 plots the annual risk exposure as a function of the number of intertipping contracts. It could be expected that contracting more intertripping services would reduce the risk. However, Fig. 4 shows a non-trivial shape where this is not necessarily the case. Note for example that $N=2$ has associated $\mathrm{M} 23.7$ compared to $\mathrm{M} £ 31.76$ when $N=7$.

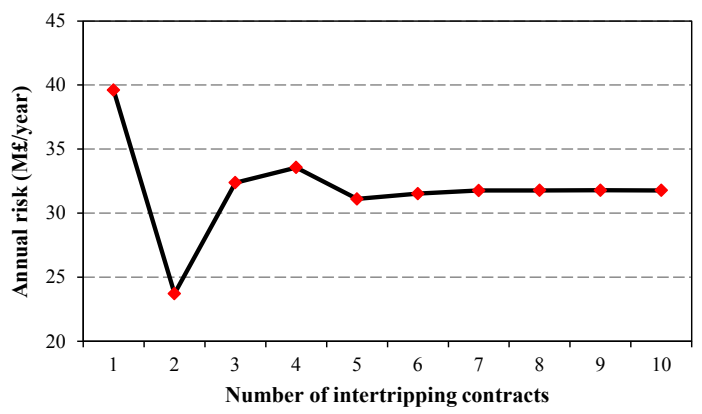

Fig. 4. Annual risk as a function of the number of intertripping contracts.

\section{2) Cost-benefit trade-off in critical operating conditions}

The analysis is now focused on understanding the reasons that have driven such unexpected results in terms of annual risk exposure. In order to do so, the operational results produced by the optimization model are inspected, hence observing important variations on risk exposure in eleven operating conditions, which in total amount to 127.7 hours.

Table II shows the data associated with those eleven operating conditions for $N$ equals to 1,2 and 7 . In particular, the table shows: demand and wind level, power transfer $(T)$, intertripping capacity and number of wind farms selected $(S)$, and risk $(X)$. The meaning of ' $C S$ ' will be explained later. It must be noted that this is a set of stressed conditions because they are characterized by bad weather, high demand and medium/high availability of wind output. In general terms, these are associated with a strong economic incentive to let wind farms in North satisfy the demand in South. However, due to the bad weather condition there is a relatively high probability of facing line outages, which may lead to higher risk exposure.

The weighted average transfer in these operating conditions (weighted by the number of hours of each of condition) is 5027, 6306 and $6391 \mathrm{MW}$ for $N$ equals to 1, 2 and 7, respectively. As the number of available intertripping contracts increases, the optimization allows larger power transfers through the transmission corridor, in order to greatly satisfy the demand in South. The utilization of the SPS, measured as the weighted average of intertripping capacity (MW) committed and the number of wind farms selected, also increases from 718.7 and 0.48 , to $1809 \mathrm{MW}$ and 3, and 1807 MW and 7 selected units, for one two and seven intertripping contracts respectively. These results show that the average utilization of the SPS notably increases with the number of intertripping schemes in order to support larger power transfer levels.

In terms of load shedding exposure, the weighted average risk in this set of critical conditions is $£ 36937.1 /$ hour, $£ 18196.3 /$ hour and $£ 86572.8$ /hour for $N$ equals to 1,2 and 7, respectively. These values show less risk aversion during critical operating conditions for the higher number of wind farms connected to the SPS.

\section{3) Partial security against SPS failures}

The columns ' $\mathrm{CS}$ ' in Table II are related to the concept of 'partial security solutions', which prevent transmission line overloads, and emergency demand curtailments, in postcontingency scenarios (include SPS failures).
TABLE II.

DATA ASSOCIATED WiTh THE CRITICAL OPERATING CONDITIONS

\begin{tabular}{|c|c|c|c|c|c|c|c|c|c|c|c|c|c|}
\hline \multirow{2}{*}{$\begin{array}{c}\text { Demand } \\
(\mathrm{pu})\end{array}$} & \multirow{2}{*}{$\begin{array}{l}\text { Wind } \\
(\mathrm{pu})\end{array}$} & \multicolumn{4}{|c|}{$\mathrm{N}=1$} & \multicolumn{4}{|c|}{$\mathrm{N}=2$} & \multicolumn{4}{|c|}{$\mathrm{N}=7$} \\
\hline & & $T(M W)$ & $\mathcal{S} \equiv(s, n)$ & $X(£ / h)$ & $C S$ & $T(M W)$ & $\mathcal{S} \equiv(s, n)$ & $X(£ / h)$ & $C S$ & $T(M W)$ & $\mathcal{S} \equiv(s, n)$ & $X(£ / h)$ & $C S$ \\
\hline 0.76 & 0.45 & 5100 & $(0,0)$ & 53671 & $\mathrm{~N}-1$ & 5100 & $(1700,2)$ & 18241 & $s_{2 / 2}^{2}$ & 6386 & $(1800,7)$ & 86610 & $S_{5 / 7}^{1}$ \\
\hline 0.76 & 0.55 & 5100 & $(0,0)$ & 53671 & $\mathrm{~N}-1$ & 5100 & $(1700,2)$ & 18241 & $s_{2 / 2}^{2}$ & 6386 & $(1800,7)$ & 86610 & $S_{5 / 7}^{1}$ \\
\hline 1.00 & 0.55 & 6200 & $(1100,1)$ & 130043 & $s_{1 / 1}^{1}$ & 6200 & $(2200,2)$ & 74221 & $s_{1 / 2}^{1}$ & 6800 & $(2380,7)$ & 83480 & $S_{5 / 7}^{1}$ \\
\hline 0.76 & 0.65 & 4700 & $(1300,1)$ & 14325 & $s_{1 / 1}^{2}$ & 5100 & $(1700,2)$ & 18241 & $s_{2 / 2}^{2}$ & 6386 & $(1800,7)$ & 86610 & $S_{5 / 7}^{1}$ \\
\hline 1.00 & 0.65 & 6400 & $(1300,1)$ & 135954 & $s_{1 / 1}^{1}$ & 6000 & $(2600,2)$ & 26584 & $s_{2 / 2}^{2}$ & 6800 & $(2380,7)$ & 83480 & $S_{5 / 7}^{1}$ \\
\hline 0.76 & 0.75 & 4900 & $(1500,1)$ & 15313 & $s_{1 / 1}^{2}$ & 5100 & $(1700,2)$ & 18241 & $s_{2 / 2}^{2}$ & 6386 & $(1800,7)$ & 86610 & $S_{5 / 7}^{1}$ \\
\hline 1.00 & 0.75 & 6600 & $(1500,1)$ & 141865 & $s_{1 / 1}^{1}$ & 6200 & $(2800,2)$ & 26584 & $s_{2 / 2}^{2}$ & 6800 & $(2380,7)$ & 83480 & $S_{5 / 7}^{1}$ \\
\hline 0.76 & 0.85 & 5100 & $(1700,1)$ & 16301 & $s_{1 / 1}^{2}$ & 5100 & $(1700,1)$ & 16301 & $s_{1 / 1}^{2}$ & 6386 & $(1800,7)$ & 86610 & $S_{5 / 7}^{1}$ \\
\hline 1.00 & 0.85 & 6800 & $(1700,1)$ & 147776 & $S_{1 / 1}^{1}$ & 6200 & $(2800,2)$ & 26584 & $s_{2 / 2}^{2}$ & 6800 & $(2380,7)$ & 83480 & $S_{5 / 7}^{1}$ \\
\hline 0.76 & 0.95 & 5100 & $(1700,1)$ & 16301 & $s_{1 / 1}^{2}$ & 5100 & $(1700,1)$ & 16301 & $s_{1 / 1}^{2}$ & 6386 & $(1800,7)$ & 86610 & $S_{5 / 7}^{1}$ \\
\hline 1.00 & 0.95 & 6800 & $(1700,1)$ & 147776 & $s_{1 / 1}^{1}$ & 6200 & $(2800,2)$ & 26584 & $s_{2 / 2}^{2}$ & 6800 & $(2380,7)$ & 83480 & $s_{5 / 7}^{1}$ \\
\hline
\end{tabular}


The notation used for the candidate solutions is $s_{\mathrm{m} / \mathrm{n}}^{l}$, where $l \in\{1,2\}$ (being 1 and 2 single or double circuit outages, respectively), and $m \in\{0, \ldots, n\}$, corresponds to the number of generators that were successfully tripped out of a total of $n$. For example, the solution $s_{5 / 7}^{1}$ is characterized by preventing post-fault overloads in the loss of a single circuit followed by the failure of two intertripping schemes (out of seven). ' $\mathrm{N}-1$ ', on the other hand, denotes the regular ' $\mathrm{N}-1$ ' security approach in which the system is resilient against a single line outage and the SPS is not utilized.

In general terms, both $\mathrm{N}=1$ and $\mathrm{N}=2$ exhibit lower risk when compared to $\mathrm{N}=7$ because they present solutions that ensure continuity of supply in case of double line outages (equivalent to a' $\mathrm{N}-2$ ' security standard), as opposed to $\mathrm{N}=7$, which protects the power system exclusively against single line outages (equivalent to a ' $\mathrm{N}-1$ ' security standard). However, it should be noted that for $\mathrm{N}=7$ the risk exposure is partially alleviated by selecting two redundant intertripping schemes that ensure continuity of supply as long as 5 out of 7 generators successfully trip in case of a single circuit outage. These results demonstrate that, under a probabilistic approach to security, redundant SPS configurations can lead the power system to larger risk exposure.

\section{4) Annual utilisation of the SPS for $N=7$}

This section looks at the whole operting year in order to provide insights into the annual utilization of the intertrippings in the case of $\mathrm{N}=7$.

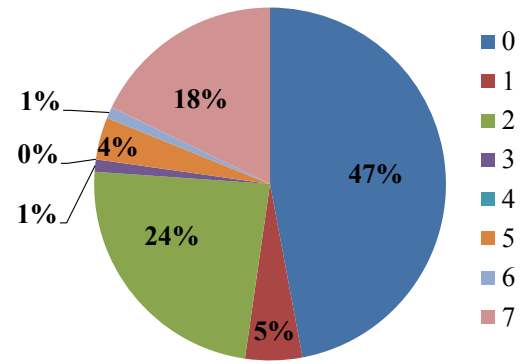

Fig. 5. Annual utilization of the intertripping contracts.

As shown in Fig. 5 almost half of the year the SPS is not operated, and the power system is run under the ' $\mathrm{N}-2$ ' security limit. When the SPS is armed, the optimization model selects only one intertripping scheme and two intertripping schemes $5 \%$ and $24 \%$ of the time, respectively. In other words, for $76 \%$ of the year contracting two intertripping schemes is sufficient to achieve optimal operational solutions, which indicates that the value of contracting further intertripping schemes is linked a restricted number of hours across the year.

\section{CONCLUSIONS}

This paper considers the system operator's decision of purchasing multiple intertripping schemes to enhance the utilization of the electricity networks. This decision is subject to an annual trade-off between capability costs, operational benefits, and the risks associated with SPS operation. The problem is formulated from a system perspective as the probabilistic minimization of intertripping capability fee payments, annual generation, protection and loss-of-load costs, for a whole operating year.

A case study based on a simple representation of the Great Britain power system is considered. The results show that, under a probabilistic security framework, contracting multiple intertripping schemes is associated with important annual benefits when compared to a traditional power system operation under the ' $\mathrm{N}-2$ ' security standard. The decision of the system operator to contract one intertripping scheme reports the largest operational savings. Nevertheless, additional intertripping contracts translated into further annual savings, until the $7^{\text {th }}$ contract. The total annual savings with respect to the ' $\mathrm{N}-2$ ' base case scenario amount to $\mathrm{M} £ 468.8$.

The presented results show that the main operational benefits are linked to stressed operating conditions characterized by bad weather, high demand and medium/high availability of wind outputs. The analysis of these critical scenarios show that the optimization model releases further transmission capacity with partial security configurations of the SPS. In particular, the SPS is configured to ensure continuity of supply upon the loss of a single circuit followed by the failure of two intertripping schemes (out of seven).

One may expect that contracting multiple intertrippings would reduce the levels of operational risk exposure. However, the inspection of the different cost components reveals that this is not certainly the case. It is possible then to conclude that, under a probabilistic framework to security, redundant SPS configurations do not necessarily reduce the operational risks, as the system operator's disposition to take risks increases due to the possibility to operate the SPS under partial security configurations.

\section{ACKNOWLEDGMENTS}

JL thanks National Grid for financially supporting his research, and FICO Xpress for proving licenses under the academic partner program.

\section{REFERENCES}

[1] PSERC, "System protection schemes: limitations, risks and management," 2010.

[2] N. Grid. Available: http://www2.nationalgrid.com/uk/services/balancingservices/system-security/intertrips/

[3] J. L. Calvo, "Balancing benefits and risks of system protection schemes," Doctor of Philosophy, Electrical and electronic Imperial College London, 2015.

[4] R. Moreno, D. Pudjianto, and G. Strbac, "Integrated reliability and costbenefit-based standards for transmission network operation," Proc. Inst. Mech. Eng. O J. Risk Reliab., November 23, 20112011.

[5] G. National, "Electricity ten year statement 2012," 20122012.

[6] P. M. Anderson and B. K. LeReverend, "Industry experience with special protection schemes," IEEE Trans. Power Syst., vol. 11, pp. 1166-1179, 1996.

[7] F. Capitanescu, J. L. Martinez Ramos, P. Panciatici, D. Kirschen, A. Marano Marcolini, L. Platbrood, et al., "State-of-the-art, challenges, and future trends in security constrained optimal power flow," Electric Power Systems Research, vol. 81, pp. 1731-1741, 8// 2011.

[8] R. Moreno, "Reliability and Cost-Benefit Based Standards for Tranmission Network Operation and Design," Department of Electrical and Electronic Engineering, Imperial College London, 2012.

[9] R. N. Allan and A. N. Adraktas, "Terminal effects and protection system failures in composite system reliability evaluation," IEEE Trans. Power App. Syst., vol. PAS-101, pp. 4557-4562, 1982. 